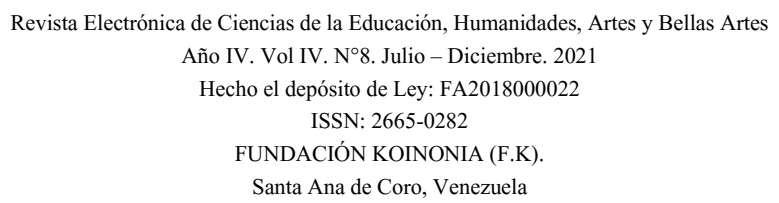

Jéssica Alexandra Concha-Maquisaca; María Isabel Álvarez-Lozano

http://dx.doi.org/10.35381/e.k.v4i8.1353

\title{
Aplicación del sistema del conocimiento a través de herramientas tecnopedagógicas en Cañar durante el COVID-19
}

\section{Application of the knowledge system through techno-pedagogical tools in Cañar during the COVID-19}

\author{
Jéssica Alexandra Concha-Maquisaca \\ jessica.concha.95@est.ucacue.edu.ec \\ Universidad Católica de Cuenca, Cañar \\ Ecuador \\ https://orcid.org/0000-0002-5215-7494 \\ María Isabel Álvarez-Lozano \\ mialvarezl@ucacue.edu.ec \\ Universidad Católica de Cuenca, Azogues \\ Ecuador \\ https://orcid.org/0000-0001-8029-1933
}

Recepción: 15 de marzo 2021

Revisado: 15 de mayo 2021

Aprobación: 15 de junio 2021

Publicación: 01 de julio 2021 


\title{
RESUMEN
}

El objetivo fue identificar cuáles fueron los retos de docentes de la Educación Intercultural Bilingüe en Cañar con la aplicación metodológica del sistema del conocimiento frente a la emergencia sanitaria COVID-19 para proponer herramientas tecnopedagógicas a la continuidad del proceso de enseñanza sincrónico o asincrónico. En la metodología se empleó un análisis descriptivo no experimental, de corte trasversal recolectando los datos con una escala de Likert y sus resultados expresados mediante estadística descriptiva. Las estrategias educativas planteadas por los docentes de Educación Intercultural Bilingüe, busca responder a las políticas educativas curriculares que logren integrar la visiones y dinámicas de una cultura, para lo cual, es necesario identificar una herramienta pedagógica que prescinda de conexión permanente para estudiantes que no tienen acceso continuo a internet y que se adapte a la metodología. Modelo del Sistema de Educación Intercultural Bilingüe [MOSEIB].

Descriptores: Método de enseñanza; enseñanza multimedia; publicación educacional. (Palabras tomadas del Tesauro UNESCO).

\begin{abstract}
The objective was to identify what were the challenges of teachers of Intercultural Bilingual Education in Cañar with the methodological application of the knowledge system in the face of the COVID-19 health emergency to propose techno-pedagogical tools for the continuity of the synchronous or asynchronous teaching process. In the methodology, a descriptive, non-experimental, cross-sectional analysis was used, collecting the data with a Likert scale and its results expressed by descriptive statistics. The educational strategies proposed by the teachers of Intercultural Bilingual Education, seek to respond to the curricular educational policies that manage to integrate the visions and dynamics of a culture, for which, it is necessary to identify a pedagogical tool that dispenses with permanent connection for students who do not have continuous access to the internet and that adapts to the methodology, Model of the Bilingual Intercultural Education System [MOSEIB].
\end{abstract}

Descriptors: Teaching methods; multimedia instruction; educational publications. (Words taken from the UNESCO Thesaurus). 


\section{INTRODUCCIÓN}

El proyecto de investigación busca analizar retos y propuestas que enfrentan los docentes de la Educación Intercultural Bilingüe (EIB) en la provincia de Cañar-Ecuador en la elaboración de guías interdisciplinares, diseñados bajo la metodologia del sistema del conocimiento propia de la educacion intercultural bilingüe, en el contexto del confinamiento por la aparición de la cepa SARS COV-2 de la familia Coronavirus, la hoy denominada COVID-19 por (Organización Mundial de la Salud OMS, 2020), que logró expandirse por todos los rincones del mundo.

Según la (Organización de las Naciones Unidas para la Educación, la Ciencia y Cultura UNESCO, 2020), las instituciones educativas a partir de marzo del 2020 cerraron sus puertas para adentrarse en el confinamiento y alrededor de 1.37 mil millones de estudiantes se vieron gravemente afectados, esto obligó a 60,2 millones de docentes innovar estrategias metodológicas para continuar con el proceso de enseñanza aprendizaje, las poblaciones viéndose gravemente afectadas sobre todo las más vulnerables de las zonas rurales y urbanas sin acceso a internet ni dispositivos tecnológicos para asistir a sus clases sincrónicas o asincrónicas.

Por otro lado, (Beltrán et al. 2020), señala que esta pandemia en España y en el mundo "marcará un antes y un después en el ámbito de la educación sobre todo en la crisis de la tecnología de quinta generación" se ve esto reflejado por la falta de recursos públicos tanto económicos-tecnológicos destinados a la educación, donde los docentes no estaban preparados ni tenían competencias en TIC.

En marzo del 2020 el Ministerio de Eduduación del Ecuador dispone que, debido a la pandemia la suspensión presencial de clases en todas las Instituciones Educativas del territorio nacional, de los diferentes sostenimientos, adicionalmente sitúa el sistema de teletrabajo al personal administrativo y docente. En consecuencia al incremento de casos confirmados el ministerio, deroga el mencionado acuerdo y en abril adiciona en la primera disposición general que las Instituciones Educativas (IE) podrán seguir con el 
desarrollo de clases de manera virtual mediante plataformas virtuales como Teamsss, Zoom, WhatsApp, Messenger, entre otras o a través de guías de interaprendizaje.

En efecto para poder seguir con el proceso de enseñanza aprendizaje, la (Secretaria del Sistema de Educación Intercultural Bilingüe SESIB, 2019), y el Distrito Educativo 03D02 Cañar, Tambo y Suscal, acuerdan que los 202 docentes que forman parte de la EIB, elaborar guías de auto e interaprendizaje basadas en el Modelo del Sistema de Educación Intercultural Bilingüe [MOSEIB], para 2727 estudiantes, surgiendo la problemática para la mayoría de docentes indígenas e hispanohablantes que no conocen la estructura de la metodología, propia de la los pueblos originarios del Ecuador.

Adicionando el déficit en conocimientos en el uso de TIC para elaborar guías y recursos interactivos para que los estudiantes desarrollen de forma autónoma o acompañada de los padres de familia, (Quero-Ramones \& Madueño-Madueño, 2006), considera que, las TIC proporciona resultados significativos, se constituye en un medio poderoso didáctico para crear imágenes, collage, búsqueda de información (textos, vídeos, audios) que responden a los saberes culturales y bilingüismo de cada pueblo. En función a lo establecido se busca identificar cuáles fueron los retos de docentes de la Educación Intercultural Bilingüe en Cañar con la aplicación metodológica del sistema del conocimiento MOSEIB frente a la emergencia sanitaria COVID-19 para proponer herramientas tecnopedagógicas útiles en el diseño de guias interactivas en el proceso de enseñanza sincrínico y asincrónico.

\section{Referencial teórico}

En el contexto educativo (Rodríguez-Pérez, 2016), sostiene que la implicación de los docentes para presentar propuestas didácticas con el uso de TIC y que sean motivantes e innovadoras para su aplicación, en efecto, se debe considerar como un modelo para viabilizar el sistema del conocimiento. Sin embargo, en las comunidades educativas donde se desarrolla un proceso de enseñanza en base al sistema del conocimiento se pueden aplicar herramientas tecnopedagógicas, sobre todo en la enseñanza de otro 
idioma como es el kichwa. Desde esta visión la metodología de la educación intercultural bilingüe es un sistema de información y creación del conocimiento, este modelo junto a la tecnología permite gestionar, analizar y organizar el conocimiento en sus etapas o procesos de desarrollo, a su vez, ¿cómo se logra dicho conocimiento? Precisamente, haciendo una buena planificación del contenido con pedagogía y tecnología.

Es importante resaltar un estudio realizado en España en el que sostienen (Sefo et al. 2017), que el uso de las tecnologías es inevitable tanto en docentes como en estudiantes, así mismo, manifiestan que entre docentes es difícil trabajar con los mismos criterios pedagógicos centrados en la innovación educativa, puesto que, no todos tienen el mismo nivel de dominio en el uso de las tecnologías. Aquí se muestra, que los docentes demandan de una actitud de formación en base a sus necesidades y conocimientos. Sin embargo, se requiere de una formación práctica y menos teórica, lo que implicaría en el profesorado aprender ciertas habilidades con el uso de manuales y recursos en red.

Los retos y propuestas que enfrentan los docentes de la EIB en América Latina y el Caribe vienen desde la década de los 40, así (Cortina, 2014), contempla que la educación bilingüe en México y en otros países del Sur de América se enmarca en ejecutar un currículo arraigado a los saberes y conocimientos de los pueblos originarios propios de su localidad. La educación intercultural centra en superar la colonialidad histórica marginal que atravesaron los pueblos indígenas, mediante el desarrollo de conocimientos andinos y occidentales con el respeto de la diversidad social. Donde (Vernimmen-Aguirre, 2019), deduce que la EIB en Ecuador se refiere más a políticas públicas plurinacionales con direcciones a una multiculturalidad que intercultural.

Con base en el desarrollo económico, social, cultural y político en los países desarrollados surgen criterios de equidad para que todos los actores educativos tengan acceso a una educación integral de calidad sin distinción alguna. Destaca la (Organización Internacional del Trabajo OIT, 2009), los derechos de los pueblos originarios indígenas a mantener una educación intercultural sin discriminación con pertenencia cultural y como medio de comunicación su lengua materna. La interculturalidad inicia con la capacidad 
del reconocimiento, respeto y diálogo de diversas culturas en un territorio (Krainer et al. 2017), en tanto perfecciona su propia identidad y respeto los demás.

Mediante las estrategias educativas planteadas por el sistema bilingüe de Chile, busca responder a las políticas educativas curriculares en su diversidad cultural, pero aún se halla en un campo educativo deficiente donde el docente no logra integrar la visiones y dinámicas de una cultura (Fernández-Droguett, 2005).

Los Centros Educativos Comunitarios Interculturales Bilingüe (CECIBs) de Ecuador han sufrido cambios abruptos a partir de la ejecución de la Asamblea Constituyente 2008, el desempeño del docente bilingüe tiene que, promover aprendizajes comunitarios holísticos e integral mediante la aplicación del MOSEIB, con el objetivo de promover estudiantes justos, innovadores y solidarios valorando sus conocimientos ancestrales y el respeto a la Pachamama (Madre Tierra).

La educación formal en las comunidades de educación intercultural bilingüe (EIB) en el Ecuador, de acuerdo con (Rodríguez et al. 2018), parte de saberes comunitarios, impulsado por líderes y movimientos indígenas en base a métodos colaborativos preservando la cultura de grupo, donde la interrelación con el otro debe producir un interaprendizaje que conlleve a una convivencia armónica.

Por consiguiente, (Granda-Merchán, 2017), señala que a partir del gobierno de la Revolución Ciudadana los postulantes a docentes del Quiero ser maestro accedieron a un nombramiento dentro de los CECIBs y a las Unidades Educativas Comunitarias Interculturales Bilingüe UECIBs desconociendo la metodología, idioma, saberes culturales, entre otras que infiere así a la autonomía educativa.

A su vez, la Universidad Nacional de Educación UNAE a partir del 2015 forma docente con licenciatura en Ciencias de la Educación Intercultural Bilingüe perfil intercultural de carácter participativa, investigativa, innovadora para edificar una sociedad equitativa y del buen vivir (Sumak Kawsay). Como señala (Rodríguez et al. 2018), el Buen vivir debe generar "dentro sistema educativo un acercamiento entre los conocimientos cientistas, basados en procesos de experimentación y validación o de aplicación tecnológica, y los 
ancestrales, con el conocimiento de la agricultura, la espiritualidad, la ritualidad y la gastronomía, entre otros saberes" (p.580) con una calidad de vida y muerte digna. No obstante, dentro de la EIB se mantienen docentes que iniciaron su labor educativo con título de secundaria o profesor bilingüe, que hoy en día les dificulta ser activos en la era digital con el uso y aplicación de las TIC, en consecuencia, de ser aplicado al diseño de guías de auto e interaprendizaje, asume así propuestas por técnicos pedagógicos bilingües con capacitaciones continuas a docentes para intentar cerrar brechas tecnológicas y educativas más aún en la fecha donde se desarrolla un retraso educativo producto del COVID-19 especialmente para los sectores de escasos recursos económicos que carecen de servicios digitales a sabiendas que, los discentes tienen derecho a continuar con sus estudios gratuitos.

\section{Metodología del Sistema del Conocimiento}

Los modelos curriculares de la EIB aplicados en los diferentes países de Latino América en primera instancia son la transición de conocimientos mediante dominio y uso de la lengua materna como recurso pedagógico de los docentes (Fajardo-Salinas, 2011). En este sentido, fue en el año 2008 que la Constitución de la República del Ecuador mediante la Asamblea Nacional, plantea a la educación como prioridad nacional y en el 2016 se realiza la reforma de la Ley Orgánica de Educación Intercultural con visión intercultural tanto para currículo intercultural y bilingüe.

En el Ecuador la estructura del Sistema Educativo Intercultural Bilingüe, centra una metodología constructivista que propone innovaciones con énfasis tecno-pedagógico, establece la metodología del sistema del conocimiento para el desarrollo del desempeño pedagógico, instrumento que se enlaza al seguimiento de los aprendizajes de los estudiantes y en alcanzar el logro del dominio participativo en el contexto familiar y comunitario. Por ende, el alcance constructivista interacciona al docente-estudiante para crear recursos necesarios y ser aplicables en la vida cotidiana (Ortiz-Granja, 2015) 
El sistema del conocimiento como proceso de enseñanza - aprendizaje en la EIB propende de una interacción entre docente-alumno y alumno-alumno. Esta metodología complementada con herramientas tecnopedagógicas genera la posibilidad de adaptar la información de acuerdo a las necesidades, características de los estudiantes y fortalecer la calidad educativa intercultural bilingüe. Los procesos educativos según (RamosCerdas, 2017), no deben ser únicamente para administrar información sino ir más allá a satisfacer las necesidades e interese de la comunidad o usuarios de la información.

Por consiguiente, la EIB basa su metodología de Sistema del Conocimiento como planificación microcurricular las guías de interaprendizaje, que es definida por la Secretaría del Sistema de Educación Intercultural Bilingüe SESEIB (2019) como "instrumento microcurricular diseñado por el docente a través de la utilización de la metodología del sistema del conocimiento, en una serie de recursos y estrategias de manera secuencial y dosificado para facilitar el proceso de interaprendizaje" (p.29), en el cual las actividades designadas a los estudiantes se correlacionan para lograr el objetivo planteado. La estructura de la metodología centra en 4 fases del conocimiento a su vez relaciona con herramientas tecnopedagógicas que requiera utilizar el docente:

Fase I. Dominio del conocimiento con fines de reconocimiento y conocimiento de contenidos esta fase subdivide en 5 sub fases:

Sub fase 1.1. Sensopercepción; Se realiza el enganche de contenidos, despertando el interés de los estudiantes mediante los sentidos a través de imágenes, videos, podcast, infografías y otras, para organizar ideas y pizarrones es el caso Padlet, Miro, Mural, Jamboard.

Sub fase 1.2. Problematización; Se establecen preguntas hipotéticas que conlleva a conocer los saberes previos, que da origen al punto de partida donde se crea la necesidad de investigación, se puede diseñar preguntas abiertas en Quizizz, Google Forms. LiveWork, Plikers.

Sub fase 1.3. Contenido científico; se enlista el contenido científico de los saberes y conocimientos a desarrollar inclinados con los conocimientos andinos, estos son propios 
de cada pueblo originario, reforzados por los saberes culturales de los Yachak, taytas y mamas de cada comunidad educativa y sin obviar los conocimientos occidentales con base a investigaciones científicas. Los temas a desarrollar se pueden presentar en Google Suite, Office Suite, llove pdf, Genially, Canva, Powtoon, Prezi, Visme, y otros.

Sub fase 1.4. Verificación; refiere a emitir respuestas de las preguntas de problematización con criterios irrefutables después de haber enriquecido el conocimiento en la sub fase de contenido científico. Así pues, describimos para evaluaciones del aprendizaje a Kahoot, Edpuzzle, Mentimeter, Quizlet, Piar deck, Quizizz.

Sub fase 1.5. Conclusión; se determina conocimientos creados y recreados para la valoración y validación de los mismos que pueden ser sintetizados en organizadores gráficos, gráficos de correlación, mapas conceptuales, maquetas, cuadros sinópticos, mentefactos, matrices, pirámides, espinas de pescado y otros. De ahí que Eduteka, Lucychart, Coggle, Mindomo, Popplet, Creately, Pixilart, Inspirartior proporciona ambientes colaborativos y didácticos.

Fase II. Aplicación; El estudiante reproduce y produce lo aprendido y aplica los conocimientos nuevos a la vida practica a través de los saberes y conocimientos mediante trabajos prácticos, colaborativos (minga), presentaciones (videos, audios afiches, trípticos, gráficos, trabajos en línea. Educandy, Educaplay, Wordwall, Metaverse, PRG (role playing game) herramientas básicas para la Gamificación de los saberes.

Fase III. Creación: Coloca en juego la imaginación de los estudiantes de nuevas situaciones, recreando-creando los saberes y conocimientos, se utiliza este espacio para tener la conexión del triángulo educativo con la Pachamama fortalecido con la cosmovisión andina a realizar actividades ejemplificadas, como, prácticas culturales en las tierras, creación de poemas, ensayos, maquetas, creación de materiales concretos, y artesanías con materia prima reciclable.

Fase IV. Socialización: los estudiantes comparten sus productos (conocimientos) con la sociedad mediante: mesas redondas, exposiciones, ferias, casa abierta, concursos, paneles, visita a museos arqueológicos, bibliotecas, Yachak, chakras, intercambio de 
riquezas culturales entre comunidades, juegos tradicionales. Se puede mencionar a Youtube, Redes sociales (WhatsApp, Facebook, Instagram, TikTok), Podcast útiles para la socialización de los conocimientos desde los estudiantes.

\section{Herramientas tecnopedagógicas}

A lo largo del desarrollo educativo se evidencia el acompañamiento de las herramientas tecnopedagógicas como estrategia pedagógica, desde una enseñanza tradicional hacia un aprendizaje más constructivo, sea ésta sincrónico o asincrónico así indican Castro et al. 2007), que las tecnologías "mejoran la manera de producir, organizar, difundir, controlar el saber y acceder al conocimiento" (p. 220). Según (Santos-Rego, 2017), las TIC siempre se usaron en el proceso de enseñanza aprendizaje, pero a partir de la creación de los Servicios de Comunicación Personal (PCs) se buscan métodos para mejorar la calidad educativa que hoy en día forma parte de las actividades curriculares y extracurriculares acorde al entorno social.

De acuerdo con (Maureen \& Glenn, 2000), el desafío de hoy en el mundo entero es hacer uso de la tecnología en el contexto de la educación y en sus diferentes eventos, es oportuno la innovación del docente. Las herramientas tecnopedagógicas transforman de acuerdo con sus ventajas: La motivación desde un aprendizaje atractivo y divertido; El interés, los recursos videos, audios, gráficos, textos y ejercicios interactivos refuerzan la comprensión multimedia; Cooperación, permite realizar trabajo o proyectos en común entre alumnos y otros colaboradores; Iniciativa y creatividad; autonomía, a través de canales el estudiante puede encontrar mucha información, solo debe seleccionar. Una de las herramientas es el eXe-Learning que brinda a los estudiantes la facilidad de poder continuar con su proceso de aprendizaje de manera asincrónica más aún diseñados en la lengua de la nacionalidad, además refuerza las aulas virtuales que necesitan de acceso permanente a internet.

Desde el punto de vista de (Karatas et al., 2017), la integración de la tecnología como herramientas principales para asistir una educación interdisciplinar a distancias no solo 
con contenidos didácticos, sino con el entorno comunitario que facilita innovar estrategias tradicionales con nuevos horizontes de entender la educación por parte de los docentes.

\section{METODOLOGÍA}

Para la ejecución del proyecto se empleó un análisis cuantitativo no experimental, según el cual, los resultados se expresan en variables de estadistica descriptiva y se caracteriza por identificar los sucesos ocurridos. Por otra parte, la información fue recolectada una sola vez, en un tiempo determinado, conviertiéndose en un estudio de tipo tranversal.

El estudio se realizó en la provincia de Cañar, Distrito Educativo 03D02 Cañar, Tambo y Suscal. Este distrito está constituído por 47 unidades educativas comunitarias interculturales bilingües con una poblacion total de 202 docentes. El diseño de la muestra es consecutivo no probabilístico. La muestra está integrada por todos los docentes que voluntariamente desearon participar del estudio. Se obtuvo 149 respuestas que representan el $70 \%$ de la población.

Para dar cumplimiento a la aproximación cuantitativa se empleó la técnica de la encuesta. Este instrumento se remitió mediante formularios Microsoff Forms siguiendo las recomendaciones para obtener resultados automáticos de la población. El instrumento cuantitativo está estructurado por un total de 22 ítems diseñados en la escala de Likert, de acuerdo con (Matas, 2018) este instrumento el encuestado emite una informacion de acuedo o desacuerdo sobre las preguntas que están agrupados en tres secciones. La primera aborda el perfil del docente, la segunda se ocupa de los desafíos que, durante la pandemia, han sorteado los docentes al aplicar la metodología del sistema del conocieminto propia de los pueblos originarios del Ecuador mediante herramientas tecnopedagógicas y, la tercera, presenta las propuestas que han estructurado los docentes frente a la situación de la emergencia sanitaria. El análisis de fiabilidad de estas tres dimensiones obtuvo un alfa de Cronbach de 0,748 equivalente a un buen nivel según el software estadisticos SPSS. 
Los resultados se presentan mediante estadísticos descriptivos mediante la medida de tendencia central del promedio o media $(\mathrm{M})$ y su desviación estándar (Des. Est.) para la escala de 1 a 5 . De este modo, el valor de 5 es el que ratifica más al ítem y el valor de 1 es el que se aleja más del ítem. Los resultados se presentan en seis tablas, a saber: 1) el perfil de los docentes, 2) desafíos bajo control del docente, 3) los desafíos del contexto, 4) herramientas de enseñanza-aprendizaje, 5) importancia del manejo de herramientas tecnopedagógicas, y 6 ) interés en participar en procesos de formación tecnopedagógicos.

\section{RESULTADOS}

Los resultados presentan los datos del perfil de los docentes y después presentan los desafíos que han sorteado los docentes al aplicar la metodologia del sistema del conocimiento propia de los pueblos originarios del Ecuador mediante herramientas tecnopedagógicas empleadas desde que inició la emergencia sanitaria por el COVID-19.

\section{Perfil de los docentes}

En la Tabla 1 se presenta los datos que describen a los docentes según el género, edad y título profesional. De acuerdo al género, el $55,7 \%$ son mujeres y la diferencia son hombres. En lo que respecta a la edad, la mayoría de docentes se encuentra en los grupos etarios de 31-40 años (43,6\%) y 41-50 años de edad (27,5\%). En lo que respecta al título profesional, se advirtió que la mayoría de docentes $(69,1 \%)$ tiene una formación de tercer nivel en educación, mientra que, en otros casos, suguiere que existen titulaciones afines como el profesor de segunda enseñanza $(15,4 \%)$, en menor medida se hallan los ingenieros, tecnólogos y otros. 
Tabla 1.

Perfil de los docentes participantes en el estudio $(\mathrm{N}=149)$.

\begin{tabular}{llcc}
\hline & & Frecuencia (N) & Porcentajes (\%) \\
\hline \multirow{2}{*}{ Género } & Masculino & 66 & 44,3 \\
& Femenino & 83 & 55,7 \\
& & & 5,4 \\
Edad & $30-30$ años & 8 & 43,6 \\
& $31-40$ años & 65 & 27,5 \\
& $41-50$ años & 41 & 22,1 \\
& $51-60$ años & 33 & 1,3 \\
& 61 o más años & 2 & 6,7 \\
& & & 69,1 \\
Título profesional & Tecnología & 10 & 4,0 \\
& Lrofesor de la Educación & 103 & 15,4 \\
& Otros & 6 & 4,7 \\
\hline Total & & 7 & 100,0 \\
\hline
\end{tabular}

Fuente: Encuesta.

\section{Desafíos tecnopedagógicos}

A continuación, se presentan los desafíos que han tenido los docentes al aplicar la metodologia del sistema del conocimiento propia de los pueblos originarios del Ecuador mediante herramientas tecnopedagógicas empleadas, desde que inició la emergencia sanitaria por el Covid-19. Todos los resultados expresan la media (M) o promedio de la escala 1 a 5 , según la cual mientras más se aproximan a 5 están más de acuerdo con que lo expresado en el ítem ocurre con mayor frecuencia. Estos resultados se presentan en cinco tablas: 1) desafíos bajo control del docente, 2) los desafíos del contexto, 3) 
herramientas de enseñanza-aprendizaje, 4) importancia del manejo de herramientas tecnopedagógicas, y 5) interés en participar en procesos de formación tecnopedagógicos. En la Tabla 2 se observan los desafíos internos, es decir, aquellos que el docente debe tenerlos bajo su control. El ítem con el que se encuentran mayormente de acuerdo es que los docentes consideran que es indispensable capacitarse de forma continua sobre el manejo de herramientas tecnopdagógicas $(M=4,39)$ y, otro ítem muy marcado, es que durante la pandemia el docente tuvo que aprender recursos tecnopedagógicos ( $M=4,22)$. Lo que menos hacen los docentes es elaborar guías con la metodología del Sistema de Conocimiento en entornos colaborativos en línea con otros docentes $(M=3,87)$.

\section{Tabla 2.}

Desafíos bajo el control de docente.

\begin{tabular}{|c|c|c|c|}
\hline Ítems & $\mathrm{N}$ & Media (M) & Desv. Est. \\
\hline Conocía Ud. la aplicación de la metodología & 149 & 3,91 & ,825 \\
\hline $\begin{array}{l}\text { Durante la pandemia Ud. tuvo que aprender recursos } \\
\text { tecnopedagógicos. }\end{array}$ & 149 & 4,22 & ,715 \\
\hline $\begin{array}{l}\text { Es indispensable para Ud. la capacitación continua sobre el manejo } \\
\text { de herramienta tecnopedagógicas }\end{array}$ & 149 & 4,39 & ,685 \\
\hline Utiliza herramientas tecnopedagógicas & 149 & 3,93 & ,831 \\
\hline $\begin{array}{l}\text { Elabora guías con la metodología del Sistema de Conocimiento en } \\
\text { entornos colaborativos en línea con otros docentes. }\end{array}$ & 149 & 3,87 & ,857 \\
\hline
\end{tabular}

Fuente: Encuesta.

Los desafíos externos son aquellos que están en el contexto del trabajo del docente, es decir, son aquellos que no puede controlarlos directamente, en tal sentido, se aluden a estudiantes, a la institución y al currículo. En la Tabla 3 se presentan estos resultados. Principalmente, los docentes creen que los estudiantes se conectan a clases virtuales y que asisten a retirar las guías, sin embargo, la media no pasa de 3,69 sobre la escala de 
5. A este ítem le sigue el que manifiesta que los contenidos de los textos coinciden con las unidades del área $(\mathrm{M}=3,28)$, sin embargo, se observa una tendencia hacia la neutralidad. En lo que respecta a los valores negativos, se observa que la institución educativa dispone de recursos económicos para la impresión de guías, aspecto con que que prácticamente no están de acuerdo $(M=1,74)$.

\section{Tabla 3.}

Desafíos externos al docente

\begin{tabular}{lccc}
\hline Ítems & $\mathrm{N}$ & Media(M) & Desv. Est. \\
\hline Los estudiantes tienen acceso a internet. & 149 & 3,08 & 1,088 \\
$\begin{array}{l}\text { Los estudiantes se conectan a clases virtuales / asisten a retirar las } \\
\text { guías }\end{array}$ & 149 & 3,69 & 1,046 \\
$\begin{array}{l}\text { La institución educativa dispone de recursos económicos para la } \\
\text { impresión de guías. }\end{array}$ & 149 & 1,74 & 1,054 \\
$\begin{array}{l}\text { Los contenidos de los textos coinciden con las unidades del área. } \\
\text { (149 }\end{array}$ & 3,28 & 1,047 \\
\hline
\end{tabular}

Fuente: Encuesta.

En la Tabla 4 se presentan los resultados de las herramientas que más emplearon los docentes para el proceso de enseñanza-aprendizaje. Al respecto, se observa que el ítem más marcado corresponde al uso frecuente de la mensajería a través de dos servicios de la empresa Facebook: Messenger y WhatsApp $(M=4,19)$. En menor medida se encuentran las guías de interaprendizaje (MOSEIB) digitales o impresas, las cuales tienen una media de 3,73 puntos. Por su parte, la Plataforma Teamsss es todavía menor en la frecuencia de uso $(M=3,56)$. La plataforma menos utilizada por los docentes es Google Classroom $(M=2,05)$. 


\section{Tabla 4.}

Herramientas de enseñanza-aprendizaje.

\begin{tabular}{lccc}
\hline Ítems & $\mathrm{N}$ & Media(M) & Desv. Est. \\
\hline Plataforma de video conferencia Teams. & 149 & 3,56 & 1,353 \\
Messenger-WhatsApp. & 149 & 4,19 &, 906 \\
Google Classroom. & 149 & 2,05 & 1,319 \\
Guías de interaprendizaje (MOSEIB) digitales o impresas & 149 & 3,73 & 1,143 \\
\hline
\end{tabular}

Fuente: Encuesta.

En la Tabla 5 se presentan los resultados vinculados a la importancia del manejo de herramietnas tecnopedagógicas, en ellas se observa que existen promedios muy altos y similares pues todos bordean el valor de 4,5 puntos en la escala de 5 puntos. La mayoría considera que es muy importante el uso de las heramientas de Microsoft y herramientas colaborativas $(M=4,48)$ y el manejo de audiovisuales y pizarras virtuales $(M=4,46)$, de cerca, les sigue el criterio de que es importante el manejo de actividades interactivas 0 de Aulas Virtuales / Exe- Learning.

Tabla 5.

Importancia del manejo de herramientas tecnopedagógicas.

\begin{tabular}{lccc}
\hline Ítems & $\mathrm{N}$ & Media(M) & Desv. Est. \\
\hline Manejo de Microsoft y herramientas colaborativas. & 149 & 4,48 &, 622 \\
Manejo de aulas virtuales/ eXe-Learning. & 149 & 4,42 &, 649 \\
$\begin{array}{l}\text { Manejo en el diseño de actividades interactivas (infografías, mapas } \\
\text { conceptuales entre otros) }\end{array}$ & 149 & 4,42 &, 670 \\
Manejo de audiovisuales y pizarras virtuales. & 149 & 4,46 &, 652 \\
\hline
\end{tabular}

Fuente: Encuesta. 
En la Figura 1 se presentan los resultados del nivel de acuerdo con el que se encuentran los docentes con respecto a recibir capacitación que vincule a la tecnología con la pedagogía. En efecto, el $65 \%$ se encuentra muy de acuerdo en recibir este tipo de capacitación y el 33\% señala estar de acuerdo. Únicamente el 2\% manifestó una opinión neutral.

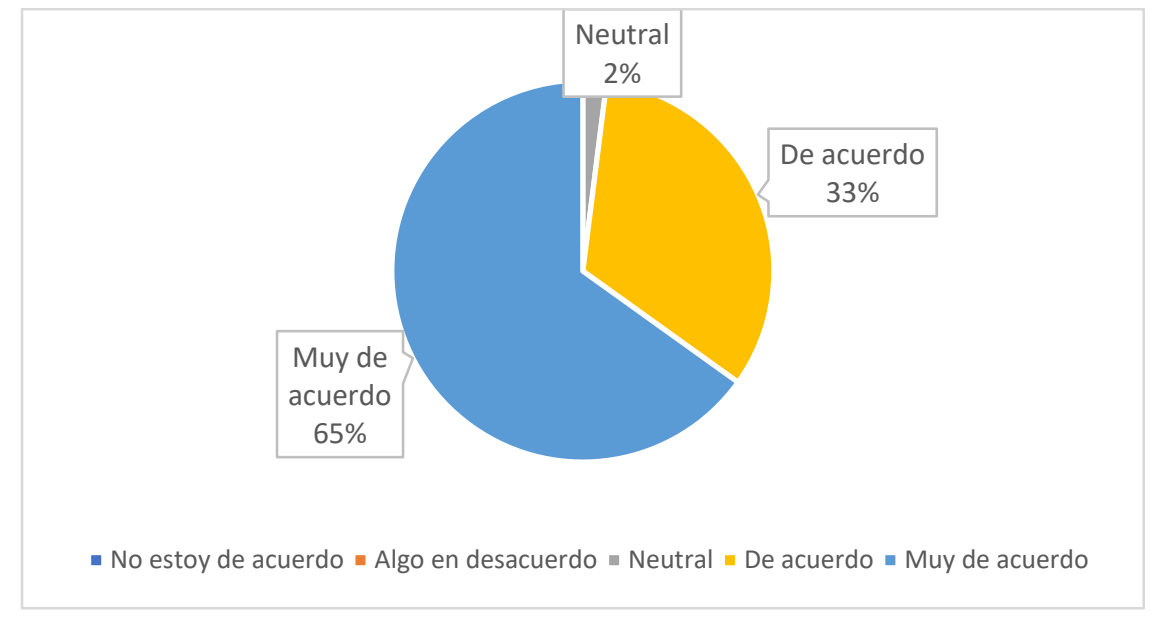

Figura 1. Nivel de acuerdo de los docentes para recibir capacitación que combina la tecnología y la pedagogía.

Elaboración: Los autores.

En definitiva, se demuestra que los desafíos bajo control del docente ha sido capacitarse en el manejo de herramientas tecnopedagógicas, sin embargo, la situación a nivel contextual muestra problemas. Aunque los estudiantes se contectan a clases virtuales 0 reciben los módulos, se observan limitantes de carácter económico, tanto para el estudiante que no tiene dispositivos e internet como para la institución que ni siquiera puede imprimir las guías para los estudiantes. En lugar de emplear las guías del MOSEIB o la plataforma Teams, la mayoría de los docentes emplea los servicios de Facebook como Messenger y WhatsApp. Un punto a considerar es el contenido de los textos que utilizan de eduación intercultural no coiciden en su totalidad con las unidades de aprendizaje. Los docentes están completamente de acuerdo en que es importante 
manejar herramientas tecnopedagógicas. Por último, la mayoría de ellos están de acuerdo en participar en procesos de formación tecnopedagógica.

\section{PROPUESTA}

El mayor desafío para los docentes han sido capacitarse, frente a las pocas oportunidades que han existido, los docentes han terminado por adoptar herramientas que no están pensadas para el desarrollo pedagógico. En efecto, han utilizado las redes sociales para enseñar a sus estudiantes, sin considerar los elementos distractores que suponen estos aplicativos. Los docentes ven como una limitación la falta de conexión de todos los estudiantes, así como la ausencia de recursos de parte de la institución para disponer de guías impresas para los estudiantes. Frente a esta situación, se plantea como un complemento a la plataforma Teams, el uso de un aula virtual que prescinde de conexión permanente. Concretamente, la propuesta plantea subir el material a un aula virtual que requiera una descarga de archivos y que funcione tanto en dispositivos móviles como en las computadoras.

Al respecto, se ha considerado oportuno emplear el software libre que pone a disposición eXe-Learning. Esta organización ha creado un dispositivo para el aprendizaje asíncrono justamente para compensar las limitaciones que tienen millones de estudiantes que no pueden participar activamente en el aprendizaje sincrónico. En este dispositivo se pueden contextualizar las guías de los docentes, basadas en la metodología del sistema del conocimiento MOSEIB para que el estudiante pueda acceder a ellas y navegar en la información ahí provista. El docente pude subir textos, imágenes, actividades interactivas, galerías o videos. Trabaja de manera similar a las aulas virtuales (Ej. Moodle), pero a diferencia de éstas, una vez que el estudiante se ha descargado la información en su dispositivo, puede desconectarse de Internet. A continuación, se observan las posibilidades del trabajo asíncrono sin conexión a Internet. 
Revista Electrónica de Ciencias de la Educación, Humanidades, Artes y Bellas Artes Año IV. Vol IV. N 8 . Julio - Diciembre. 2021

Hecho el depósito de Ley: FA2018000022 ISSN: $2665-0282$

FUNDACIÓN KOINONIA (F.K)

Santa Ana de Coro, Venezuela

Jéssica Alexandra Concha-Maquisaca; María Isabel Álvarez-Lozano

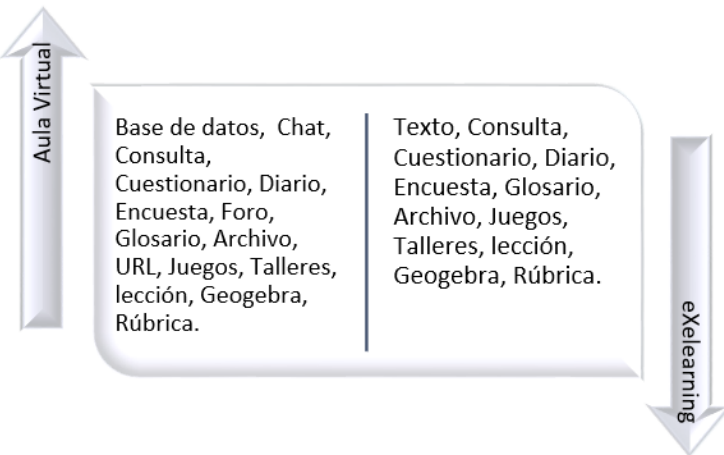

Figura 2. Comparación entre un Aula virtual y la plataforma eXe-Learning. Elaboración: Los autores.

Como se observa en la Figura 2, únicamente dos herramientas que se emplean en vivo no se utilizan dentro de esta plataforma y se refiere concretamente al Chat y los foros que requieren de una conexión permanente en Internet. En este sentido, la plataforma que se propone como solución resulta muy funcional a los propósitos de aprendizaje planteados por el MOSEIB.

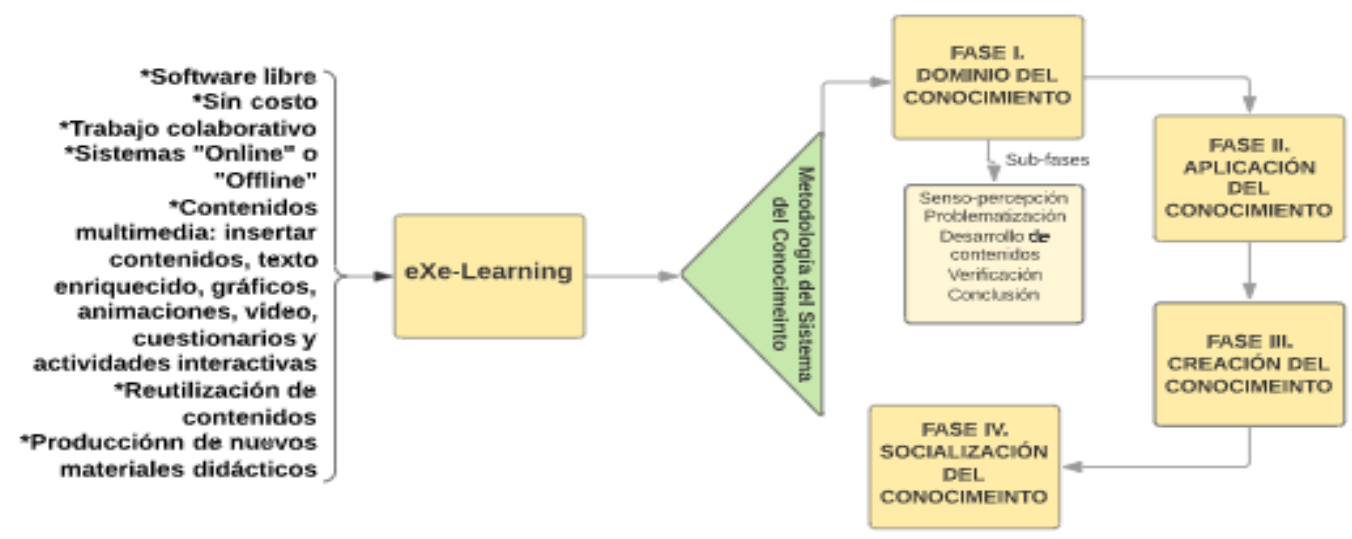

Figura 3. Integración de la metodología del sistema del conocimiento en eXe-Learning. Elaboración: Los autores. 
De todas las posibilidades de la plataforma eXe-Learning, se propone utilizar las necesarias para dar cumplimiento a las guías del MOSEIB. Por ejemplo, Figura 3. Para enseñar los saberes y conocimientos CN.EIB.69.13. se emplea textos interactivos, videos e infografías diseñadas en la lengua de la nacionalidad kichwa, cuestionario con retroalimentación y un archivo. Como se observa, no existen diferencias con el empleo de una plataforma que requiere conexión a Internet.

Pasos a seguir para la instalación de eXe-Learning:

- Ingresa a la página web de eXe-Learning: https://exelearning.net/descargas/ selecciona la opción versión portable para Windows.

- El portable INTEF-eXe-install-2.4.2 automáticamente aparecerá en descargas.

- Seleccione el archivo INTEF-eXe-install-2.4.2 de descargas con doble clic, Next, Agreen, Install, Finish. [En el desplegable de menú herramientas seleccione su idioma de preferencia(español)]. El programa estará listo para desarrollar las actividades didácticas.

- Selecciona dentro de los programas de tu computador eXe-Learning con doble clic.

- Dentro del software en se encuentran las herramientas (Añadir página, borrar, renombrar) y en la parte inferior están los Divice, que permiten añadir textos, tareas, actividades interactivas y otros contenidos.

En vista de aquello se adjunta un link ejemplificado la metodología del sistema del conocimiento en eXe-Learning: https://n9.cl/o79qb Copie el link en su sitio web y descargue la carpeta titulada eXe-Learning_1, dentro de los archivos seleccione con doble clic la opción 1er. GUIA.html. 


\section{CONCLUSIONES}

El impacto de la tecnología en la educación propende de un cuestionamiento sobre nuevas formas de enseñar y aprender, nuevas pedagogías y metodologías. Las herramientas tecnopedagógicas facilitan nuevos rumbos de innovación educativa sin prescindir el rol y la mediación docente. Sin embargo, no se debe caer en el tecnocentrismo por muy novedosa que sea su integración al proceso de enseñanza aprendizaje, hay que anclar al modelo pedagógico sin desvirtuar sus bondades.

En definitiva, se demuestra que los desafíos bajo control del docente ha sido capacitarse en el manejo de herramientas tecnopedagógicas, sin embargo, la situación a nivel contextual muestra problemas. Aunque los estudiantes se conectan a clases virtuales 0 reciben los módulos, se observan limitantes de carácter económico, tanto para el estudiante que no tiene dispositivos e internet como para la institución que ni siquiera puede imprimir las guías para los estudiantes. En lugar de emplear las guías del MOSEIB o la plataforma Teams, la mayoría de los docentes emplea los servicios de Facebook como Messenger y WhatsApp. Los docentes están completamente de acuerdo en que es importante manejar herramientas tecnopedagógicas. Por último, la mayoría de ellos están de acuerdo en participar en procesos de formación tecnopedagógica.

La implicación de los docentes para presentar propuestas didácticas con el uso de herramientas tecnopedagógicas, que sean motivantes e importantes, esto se debe tomar como modelo para viabilizar el sistema del conocimiento. Sin embargo, en las comunidades educativas donde se desarrolla un proceso de enseñanza en base al sistema del conocimiento se pueden aplicar la TIC, sobre todo en la enseñanza de otro idioma como es el kichwa. Desde esta visión el sistema del conocimiento es un sistema de información y creación del conocimiento, este modelo junto a la tecnología permite gestionar, analizar y organizar el conocimiento en sus etapas, herramienta que permite adjuntar imágenes, videos, archivos, link, cuestionarios, rúbricas y otros.

La aplicación del sistema del conocimiento mediante el uso de herramientas tecnopedagógicas como es el eXe-Learning en la pandemia, se convierte en un 
andamiaje dinámico y didáctico con enfoque socioconstructivista, importante para la construcción y aplicabilidad del conocimiento con pertinencia cultural y lingüística, enfocados en el currículo intercultural bilingüe con resultados satisfactorios, más aún en la emergencia sanitaria, resulta como una de las soluciones innovadoras de los docentes para hacer de su labor significativo, enfocados en el sector rural que no disponen del servicio continuo de internet.

\section{FINANCIAMIENTO}

No monetario.

\section{AGRADECIMIENTO}

A la Corporación Eléctrica del Ecuador y la Jefatura de Posgrados de la Universidad Católica de Cuenca por permitir el desarrollo y fomento de la investigación.

\section{REFERENCIAS CONSULTADAS}

Beltrán, J., Venegas, M. Villar-Aguilés, A., Andrés, S., Jareño-Ruiz, D., \& de-GraciaSoriano, P. (2020). Educar en época de confinamiento: la tarea de renovar un mundo común [Educating in times of confinement: the task of renewing a common world]. Revista de Sociología de la Educación-RASE. 13.92. 10.7203/RASE.13.2.17187.

Castro, S., Guzmán, B., \& Casado, D. (2007). Las TIC en los procesos de enseñanza y aprendizaje [ICT in teaching and learning processes]. Laurus, 13(23),213-234.

Cortina, R. (2014). Educación intercultural bilingüe en latinoamérica. [Intercultural Education in Latin America]. Revista Mexicana de Investigación Educativa, 19, 1318. https://n9.cl/wlnqc

Fajardo-Salinas, D. (2011). Educación intercultural bilingüe en Latinoamérica: un breve estado de la cuestión [Bilingual intercultural education in Latin America: a brief state of the art]. LiminaR Estudios Sociales y Humanísticos, 9(2), 15-29 
Fernández-Droguett, F. (2005). El currículum en la educación intercultural bilingüe: algunas reflexiones acerca de la diversidad cultural en la educación [The curriculum Intercultural Education: some reflections on cultural diversity in education]. Cuadernos Interculturales, 3(4), 7-26. https://n9.cl/k7nr2

Granda-Merchán, S. (2017). La institucionalización de la educación intercultural bilingüe en el Ecuador y su impacto en las iniciativas de educación propia. [The institutionalization of bilingual intercultural education in Ecuador and its impact on self-education initiatives Reflec]. https://n9.cl/2c5li

Karatas, I., Tunc, M., Yilmaz, N., \& Karaci, G. (2017). An Investigation of Technological Pedagogical Content Knowledge, Self-Confidence, and Perception of Pre-Service Middle School Mathematics Teachers towards Instructional Technologies. Journal of Educational Technology \& Society, 20(3), 122-132. http://www.jstor.org/stable/26196124

Krainer, A., Aguirre, D., Guerra, M., \& Meiser, A. (2017). Educación superior intercultural y diálogo de saberes: el caso de la Amawtay Wasi en Ecuador. [Intercultural higher education and knowledge dialogue: The case of Amawtay Wasi University in Ecuador]. Revista de La Educacion Superior, 46(184), 55-76. https://doi.org/10.1016/j.resu.2017.11.002

Matas, A. (2018). Diseño del formato de escalas tipo Likert: un estado de la cuestión. [Likert-Type Scale Format Design: State of Art]. Revista Electronica de Investigacion Educativa, 20(1), 38-47.

Matas, A. (2018). Diseño del formato de escalas tipo Likert: un estado de la cuestión. [Likert-Type Scale Format Design: State of Art]. Revista Electronica de Investigacion Educativa, 20(1), 38-47. https://doi.org/10.24320/redie.2018.20.1.1347

Maureen L., J. Glenn P., M. T. (2000). Inverting the Classroom: A Gateway to Creating an Inclusive Learning Environment [Invertir el aula: una puerta de entrada a la creación de un entorno de aprendizaje inclusivo]. The Journal of Economic Education, 31(1), 30-43. https://n9.cl/5qff

Modelo del Sistema de Educación Intercultural Bilingüe [MOSEIB]. https://n9.cl/4hd3In 
Organización de las Naciones Unidas para la Educación, la Ciencia y la Cultura UNESCO. (2019). Ciudadanía mundial y derechos humanos [World citizenship and human rights]. https://n9.cl/wqeqk

Organización Internacional del Trabajo OIT. (2009). Los derechos de los pueblos indígenas y tribales en la práctica [The rights of indigenous and tribal peoples in practice]. https://n9.cl/1ml63

Organización Mundial de la Salud-OMS. (2020). Alocución de apertura del Director General de la OMS en la rueda de prensa sobre la COVID-19 celebrada el 11 de marzo de 2020. [Opening speech by the Director-General of WHO at the press conference on COVID-19 held on March 11, 2020]. https://n9.cl/qejpo

Ortiz-Granja, D. (2015). El constructivismo como teoría y método de enseñanza. [Constructivism as theory and teaching method]. Sophia, 19(2), 93-110. https://doi.org/10.17163/soph.n19.2015.04

Quero-Ramones, S, \& Madueño-Madueño, L. (2006). Süchiki Walekerü: un ejemplo del uso de las TIC en escuelas indígenas, caso Wayuu [Süchiki Walekerü: an example of the use of ICT in indigenous schools, Wayuu case]. Educere, 10(34),435-442.

Ramos-Cerdas, A. (2017). Gestión del conocimiento en el proceso de docencia para instituciones de educación superior. [Knowledge management in the process of teaching for higher education institutions]. SIGNOS - Investigación En Sistemas de Gestión, 7(2), 31. https://doi.org/10.15332/s2145-1389.2015.0002.02

Rodríguez, M. N., Aguilar, J., \& Apolo, D. (2018). El Buen vivir como desafío en la formación de maestros: aproximaciones desde la Universidad Nacional de Educación del Ecuador [Good living as a challenge in teacher training: approaches from the National University of Education of Ecuador]. Revista Mexicana de Investigación Educativa, 23(77), 577-596. https://n9.cl/2tbht

Rodríguez-Pérez, N. (2016). ¿Las TIC como mediadoras en la enseñanza-aprendizaje de lenguas extranjeras? [ICT as mediators in the teaching-learning of foreign languages?]. Opción, 32(10),569-588.

Santos-Rego, M. (2017). La educación intercultural y el pluralismo religioso: propuestas pedagógicas para el diálogo [Intercultural education and religious pluralism: pedagogical proposals for dialogue]. Educación XX1, 1-4. https://doi.org/https://doi.org/10.5944/educxx1.17489 
Secretaría del Sistema de Educación Intercultural Bilingüe-SESEIB. (2019). Orientaciones Pedagocicas para fortalecer la implementación del MOSEIB. [Pedagocical Orientations to strengthen the implementation of the MOSEIB]. Ministerio de Eduación, 53(9), 1689-1699. https://n9.cl/4gdqg

Sefo, K., Granados Romero, J. M., Lázaro, M.-N., \& Fernández_Larragueta, S. (2017). La formación del profesorado para un uso innovador de las TIC: un estudio de caso en la educación obligatoria en la provincia de almería. [Teacher training for an innovative use of ICT: A case study within compulsory education in the region of Almeria]. Revista de Currículum y Formación de Profesorado, 21(4),241-258. https://n9.cl/n902lp

Vernimmen-Aguirre, G. M. (2019). Educación Intercultural Bilingüe en Ecuador: Una revisión conceptual. [Ecuadorian Intercultural Education: a conceptual]. Alteridad, 14(2), 162-171. https://doi.org/10.17163/alt.v14n2.2019.01

C2021 por los autores. Este artículo es de acceso abierto y distribuido según los términos y condiciones de la licencia Creative Commons Atribución-NoComercial-Compartirlgual 4.0 Internacional (CC BY-NC-SA 4.0) (https://creativecommons.org/licenses/by-nc-sa/4.0/). 